\title{
Predictive methodology and application in economics and finance: Volume in honor of the accomplishments of Clive W.J. Granger
}

This volume of the Journal of Econometrics is very special to us, the editors, as its publication is our way of expressing, albeit in a modest way, our unlimited admiration and respect for the 2003 Nobel laureate in economics, Clive W.J. Granger. He has served in a variety of capacities as mentor, friend, and colleague to each of us, and for that, we are profoundly grateful. Just as importantly, it is our way of signaling the fact that even though Clive has recently retired, we fully expect to see his brilliant research continue indefinitely. Without his insights and direction, we and much of the discipline would be lost. Indeed, one need simply read his opening comments in the first volume of this set, and his contribution later on with Mark Machina, to see that he indeed continues to dazzle.

One prominent theme in Granger's work has been the centrality of forecasting in time series econometrics. This follows a few distinct lines of thought. First, in the face of uncertainty over the true model, Clive has promoted the idea that models should be selected based on their forecast performance rather than based on insample fit. Second, it is not obvious that the conditional mean is the only statistic of interest (see e.g. Granger, 1969, "Prediction with a Generalized Cost of Error Function", Operations Research Quarterly, 20, 199-207; and Granger, C.W.J., 1993, "On the Limitations of Comparing Mean Squared Forecast Errors: Comment", Journal of Forecasting, 12, 651-652). In the aforementioned paper, Granger examines the combinations of loss function and data distributions that result in the conditional mean being the optimal forecast. These combinations are found to be quite restrictive, and hence restricting examination to the conditional mean is similarly restrictive. This also implies that other moments or the entire conditional density should be of interest in model selection. A number of papers in this special issue extend and make these ideas practical. Of course, Granger's papers in forecasting include many other seminal contributions, including ones discussing forecast combination, forecast model construction and selection, forecast evaluation and accuracy assessment, and forecasting in finance, for example (see e.g. Granger, C.W.J. and P. Newbold, 1986, Forecasting Economic Time Series, Academic Press: 
San Diego, as well as Ashley. R., Granger, C.W.J. and R. Schmalensee, 1980, "Advertising and Aggregate Consumption: An Analysis of Causality," Econometrica, 48, 1149-1168). For a fairly complete collection of many of Clive's most recognized papers in the areas of forecasting as well as in other areas he has worked in, see Ghysels, Swanson and Watson, 2001, Essays in Econometrics: Collected Papers of Clive W.J. Granger, Volumes I and II , Cambridge University Press: Cambridge, MA. Papers addressing the above areas in forecasting in this volume include those by Aiolfi and Timmermann; Andreou and Ghysels; Clark and West; Corradi and Swanson; Egorov, Hong and Li; Hansen; Hendry; Lee and Yang; and Marcelino, Stock, and Watson.

Related to Granger's profound work in the area of forecasting is the ubiquitous notion of Granger causality (see e.g. Granger, C.W.J., 1969, "Investigating Causal Relations by Econometric Models and Cross Spectral Methods", Econometrica, 37, 424-438; and Granger, C.W.J., 1980, "Testing for Causality: A Personal Viewpoint", Journal of Economic Dynamics and Control, 2, 329-352), a concept that is crucial in the construction of predictions, and in carrying out natural experiments in practical settings such as policy analysis. Contributions to this area of research in the volume include those by Dufour and Jouini as well as White.

Another immensely important contribution of Clive is his notion of cointegration, and the recognition that nonstationarity and cointegration arise naturally in a variety of theoretical and empirical settings (see e.g. Engle, R.F. and C.W.J. Granger, 1987, "Co-integration and Error Correction: Representation, Estimation, and Testing", Econometrica, 55, 251-276; Granger, C.W.J., 1981, "Some Properties of Time Series Data and Their Use in Econometric Model Specification", Journal of Econometrics, 16, 121-130; and Granger, C.W.J. and P. Newbold, 1974, "Spurious Regressions in Econometrics", Journal of Econometrics, 2, 111-120). The papers by Elliott and Mueller as well as Hsiao and Wang contribute to these literatures.

A different strain of Granger's work focuses on nonlinear models. He has made numerous contributions in the areas of nonlinear model specification, estimation, and prediction, for example. Additionally, he has developed and introduced a plethora of important new nonlinear models to the literature, and everyday notions such as "long memory" derive from his work. For some discussion of a very few of the developments for which his work has been instrumental, see, e.g. Granger, C.W.J. and T. Teräsvirta, 1993, Modelling Nonlinear Economic Relationships, Oxford University Press: Oxford; and Lee, T.-H., H. White and C.W.J. Granger, 1993, Testing for Neglected Nonlinearity in Time Series Models: A Comparison of Neural Network Methods and Alternative Tests, Journal of Econometrics, 56, 269-290. Chen and Fan; Gonzalo and Martinez; and Haldrup and Nielson have contributions in this area.

Of course, it must also be noted that Granger has made various contributions to our knowledge of general statistical methods (see, e.g. Granger, C.W.J., 1979, "Near Normality and Some Econometric Models", Econometrica, 47, 781-784, as well as Granger, C.W.J. and M.J. Morris, 1976, "Time Series Modelling and Interpretation", Journal of the Royal Statistical Society, A, 139, 246-257). In light of this, the volume is proud to also include a piece by T.W. Anderson. 
Finally, Granger's work in the area of financial econometrics cannot be underscored enough. From his early days working with Michio Hatanaka under Oskar Morgenstern to his recent work in long memory, he has always had many ideas that have proven crucial to our understanding of financial markets (see, e.g. Granger, C.W.J. and O. Morgenstern, 1970, Predictability of Stock Market Prices, Heath and Company: Lexington, MA, as well as Ding, Z., R. Engle and C.W.J. Granger, 1993, “A Long Memory Property of Stock Market Returns and a New Model", Journal of Empirical Finance, 1, 83-106). In this celebration of Clive, it is with great pleasure that we are able to include an important new paper in this area by Granger and Machina.

In summary, the accomplishments of Clive W.J. Granger are truly too numerous to list. What we offer here is merely a small homage to him. Little more need be said. The remainder of this introduction briefly summarizes the papers contained in the volume.

The first paper in this volume summarizes the opening conference comments made by Clive W.J. Granger. As is always the case with the written works of Clive, these comments, while concise, contain numerous important statements concerning the direction that time series econometrics will undoubtedly take over the next decade or two. Additionally, these comments include a heartfelt discussion of the events leading up to the conference, including Clive's retirement, his being awarded yet another honorary degree, and of course his finding out about the greatest honor of all-being awarded the Nobel prize in economics. As with all of Clive's written works, this is one that will undoubtedly be read numerous times by econometricians of both theoretical and applied ilk, with new treasures of information gleaned upon each additional reading.

In a second paper in the volume, Clive Granger and Mark Machina point out that virtually all nonlinear economic models with independent, identically distributed stochastic shocks and time-invariant structural parameters will generate persistent, partially predictable heteroskedasticity ("volatility clustering") in their key dependent variables. The goal of their paper is to explore ways in which the phenomenon of volatility drift or volatility clustering, of the form modeled by $\mathrm{ARCH}$ and other stochastic volatility specifications, can arise in, and in turn be modeled by, systems whose nonstochastic structure is time-invariant and whose stochastic shocks are all zero-mean i.i.d. and consequently homoskedastic. Granger and Machina present some general mechanisms and specific examples of how a timeinvariant system subject to i.i.d. shocks can exhibit volatility clustering in its key dependent variables. The authors also introduce the notion of "structural attribution" of observed volatility clustering by means of a similar but more straightforward exercise, namely the structural attribution of observed serial correlation in an economic time series.

The main objective of the Granger and Machina paper is profound as they study how volatility clustering relates to the structural (i.e. deterministic and causal) properties of a time-invariant economic system which is subject to irreducible "white noise" uncertainty, rather than as an exogenous stochastic property of a single time series variable. The paper also addresses the forecasting implications of i.i.d. shock, 
time-invariant structural forms which generate several familiar types of volatility clustering.

Marco Aiolfi and Allan Timmermann consider various measures of persistence in the (relative) forecasting performance of linear and nonlinear time series models applied to a large cross-section of economic variables in the G7 countries. They note that model instability is a source of misspecification that is likely to be particularly relevant in practice and it is therefore highly unlikely that a single model will dominate uniformly across time. If the identity of the best local model is time varying, it is implausible that a forecasting strategy that, at each point in time, attempts to select the best current model will work well. Most obviously, if (ex-ante) the identity of the best model varies in a purely random way from period to period, it will not be possible to identify this model by considering past forecasting performance across models. Similarly, if a single best model exists but it only outperforms other models by a margin that is small relative to random sampling variation, it becomes difficult to identify this model by means of statistical methods based on past performance. Unfortunately, little is known about persistence in forecasting performance, so the first part of the paper considers this question, establishing "stylized facts" by studying empirically a large cross-section of economic variables and forecasting methods. Marco Aiolfi and Allan Timmermann find strong evidence of persistence among top and bottom forecasting models, but also systematic evidence of "crossings"- where a previously good (poor) forecasting model delivers poor (good) future forecasting performance-among the linear models. Persistence in forecasting performance is related to the possibility of improving performance through forecast combinations. The authors propose a new four-stage conditional model combination method that first sorts models into clusters based on their past performance, then pools forecasts within each cluster, followed by estimation of the optimal forecast combination weights for these clusters and shrinkage towards equal weights. These methods are shown to work well empirically in out-of-sample forecasting experiments.

The paper by T.W. Anderson builds on a rich literature of estimation in econometrics, a literature which owes much to the early seminal works of Anderson, and to numerous other works by the author over the last 60 years. In this interesting paper, Anderson elucidates how to use reduced rank regression in order to provide maximum likelihood estimators of regression coefficients with linear restrictions. One novel feature of the paper is that it is shown that the maximum likelihood estimator of the restrictions can be approximated using only the eigenvectors of a matrix in the metric of the error covariance matrix. The procedures are applied to a block of simultaneous equations, and are shown to be generalizations of limited information maximum likelihood and two-stage least squares.

Most of the statistical and econometric literature of sequential change-point analysis have focused on linear regression models or linear dynamic models with weak dependence. The paper of Andreou and Ghysels analyzes historical and sequential CUSUM change-point tests for nonlinear strongly dependent processes, and in particular processes describing volatility dynamics. These tests are used to monitor the conditional variance of asset returns and to provide real-time 
information regarding instabilities in financial risk. In more detail, this paper provides the asymptotic results for the CUSUM test for strongly dependent time series and in particular for financial time series processes featuring volatility clustering. It emphasizes the use of data-driven monitoring processes, and considers the effect of sampling frequency on the local power of CUSUM tests. The latter is analyzed analytically as well as in an extensive Monte Carlo exercise. The analytical results provide evidence of some nontrivial trade-offs between relative local power and the role of sampling frequency, persistence and tails of the volatility process. The simulation analysis unfolds the finite sample properties of the CUSUM volatility change-point test and gives practical rules to choose between different type of boundaries.

The paper by Chen and Fan takes up the challenge of examining model selection amongst general models using the entire distribution. They suggest the estimation of dynamic multivariate models using a semi-parametric approach based on copulas. The paper provides methods for model selection between potentially misspecified models.

Clark and West address an issue that has been examined many times in the finance literature, for example. Namely, they consider using out-of-sample mean square prediction errors (MSPEs) to test whether a series follows a martingale difference process. The alternative of their test is linear predictability, and they show, via careful assessment of parameter estimation error that the alternative models' MSPE is expected to be greater than the null's. This important fact can lead to the alternative model being preferred in cases where its MSPE is greater than under the null.

The paper of Corradi and Swanson tackles three key issues in forecasting, namely, examining out-of-sample predictive accuracy using the entire distribution rather than a single summary statistic, the construction of test statistics that properly account for parameter estimation error and recursive or rolling estimation methods, and the development of testing methodology that allows models being compared to be (dynamically) misspecified. The paper provides methods for testing out-of-sample predictive accuracy between multiple conditional distributional models. In practice, this is of course difficult given the complexity of the possible models; however they are able to get around this by providing simple-to-use bootstrap approaches to the construction of asymptotically valid critical values in a variety of estimation contexts.

Dufour and Jouini tackle the problem of poor finite sample performance of asymptotic inference for VARs. Granger (1969) introduced the popular idea of Granger Causality testing; however, in practice for sample sizes available in macroeconomics often the number of parameters to be estimated is too large with respect to available number of observations for asymptotic approximations to result in tests with adequate size control. The authors suggest a Monte Carlo approach to construct tests that control size.

Egorov, Hong, and $\mathrm{Li}$ examine the relative values of random walk models for the interest rate versus affine models. In most examinations of the affine model for interest rates, model evaluation is done in sample. The authors here follow Granger's 
concerns about in-sample evaluation and examine the out-of-sample forecast performance of the models. They find that whilst random walk models do well for the mean, the affine model better captures other features of the forecast density.

Elliott and Mueller address the topic of unit root testing, a topic which Clive essentially pioneered in economics with his papers on nonstationarity in the 1970s and 1980s. In their paper, Elliott and Mueller begin by noting that the outcome of the most widely used unit root tests depends crucially on the initial condition, which is a somewhat disturbing fact. They continue to elucidate, in their usual clear and concise style, numerous methods for constructing unit root tests that are little affected by the initial condition. They continue along this vein by deriving an asymptotically efficient test for which the power varies as little as possible as a function of the intial condition.

Gonzalo and Martinez wonder what the shocks are that drive economic fluctuations. The answer to this question requires as a first step solving the shock identification issue. Traditionally, this has been done by decomposing the analyzed variable into unobserved permanent and transitory components. Some examples are: (1) Beveridge and Nelson decomposition, where the permanent component is a random walk and there is perfect correlation between permanent and transitory shocks due to the fact that there is only one shock, the permanent one, (2) Unobserved component models with uncorrelated components, where the permanent component is also assumed to be a random walk but its innovation is uncorrelated with the one in the transitory term. The authors propose a new identification scheme based on two aspects: (i) the long-run effect of the shock (permanent or transitory) and (ii) the size of the shock (large or small). This is done by using a threshold-integrated moving average model (TIMA) previously introduced in the literature by the authors. Such model enables them to develop a testing strategy to determine whether large and small shocks have different long-run effects, as well as whether one of them is purely transitory. In other words, the authors know, through their model, if the size of the shock matters in order to explain economic fluctuations. The paper analyzes the impulse response function of both types of shocks, and provides the asymptotic results sufficient to test if shock size matters in the long run. It also develops a new nonlinear permanent-transitory decomposition. The new decomposition shares the spirit of Beveridge-Nelson and orthogonal unobserved components decompositions, though its behavior lies somewhere between them. The results in the paper are applied to stock prices, to measure the quality of the stock market, and to GNP, to analyze the existence of asymmetries in the persistence of output shocks.

The paper of Haldrup and Nielsen tackles the issue of nonlinearity and long memory. It has now become well known that nonlinear models, like regime switching models, can generate spurious long memory. Haldrup and Nielsen propose a model that is able to nest both possibilities. This is a Markov switching model with long memory in the separate regime states. The model is motivated by the features of electricity prices in regions where physical interconnections in the exchange of electricity exist bilaterally. In this framework, the presence or absence of congestion defines in a natural way two different regimes. In each regime prices may have 
different degrees of long memory and therefore the model introduced by the authors can potentially exhibit state-dependent fractional cointegration. The regime switching model with long memory is applied to describe empirically the dynamics of electricity prices within the Nord Pol area and the paper finds that the switching model provides better forecasts (with respect to nonswitching models) for the relative prices, and it appears to be particularly successful when regime persistence is high and the post-sample observations belong to the noncongestion regime.

Bruce Hansen contributes to this special issue by focusing on the construction of interval forecasts in the face of parameter uncertainty. This is an important topic because, as noted by Hansen, forecast intervals constructed using time series models often sidestep the issue of parameter estimation. The proposed solution to this problem given by Hansen is simple, easy to apply, and yields nice results in simulation experiments. In short, the solution involves interval endpoint adjustment based on the magnitude of the asymptotic variance of the interval estimate.

It is well known that in cointegrated systems forecast must be made via equilibrium-correction models (VEqCMs). David Hendry's paper alerts us about the risk of using these models when a location shift, such as changes in equilibria, occurs. In this situation, forecasts from $\mathrm{VEqCMs}$ tend to move in the opposite direction to the data, thereby inducing forecast failure. Hendry proposes two adaptations to improve the robustness of forecasts from equilibrium-correction systems. The first is using second differences to forecast (DDD) and the second is forecasting from the differenced VEqCM (DVEqCM). The paper provides a theoretical ranking of these methods when we forecast after a location shift: the DVEqCM should dominate DDD in both mean and variance and hence in MSFE, DDD should dominate VEqCM in mean, but not necessarily in variance, so MSFE comparisons depend on the magnitudes of shifts relative to error variances, and hence DVEqCM should dominate VEqCM in mean, but not necessarily in variance or MSFE.

The paper provides the empirical example of the behavior of M1 in the UK following the Banking Act of 1984; the difficulty of outperforming "naïve extrapolative devices" when these are adaptive to precisely those location shifts that are inherent to all econometric systems is shown.

In the paper by Hsiao and Wang, the authors contribute to the huge literature generated by the idea of cointegration. They extend Hsiao's earlier results on structural modeling of cointegrating models to near-cointegrating models using the fully modified approach.

Lee and Yang examine bagging methods when one is trying to predict binary outcomes or quantiles. They show some small sample gains through the use of bagging (bootstrap averaging) forecast procedures for these applications. The focus on alternatives to the conditional mean fits with the second of Granger's ideas on forecasting.

The Marcellino, Stock and Watson paper compares, in terms of MSFE, direct versus iterated forecasts when both are based on univariate and bivariate autoregressive models. The iterated method produces more efficient parameter estimates than the direct method, but it is prone to bias if the one-step ahead model is misspecified. This produces a theoretical ambiguity in terms of relative efficiency 
that depends heavily on the unknown population best linear projection. Because of that, the theoretical literature tends to conclude that the robustness of the direct forecast to model misspecification makes it a more attractive procedure than the bias-prone iterated forecast. In this paper, the authors maintain that this result is not so clear and that the issue of which method to choose is an empirical one. For that reason, they undertake a large-scale empirical comparison of iterated versus direct forecast using data on 170 US macroeconomic monthly time series variables from 1959 to 2002. Their main finding is that iterated forecasts tend to have smaller MSFEs than direct forecasts, particularly if the iterated forecasts are computed using AIC lag length selection. Therefore, they conclude that it appears, in practice, that the robustness and bias reduction obtained using direct forecasts does not justify the price paid in terms of increased sampling variance.

White contributes to this volume by including a paper that gets at causality via examination of natural experiments. His paper parallels closely the classical treatment effects literature, with a variety of new wrinkles added due to the use of time series data, and due to his ability to concisely bring together notions used in cross-sectional econometrics with those used in time series econometrics. $\mathrm{He}$ discusses testing and estimation of causal effects.

\section{Editorial collaborators}

The following individuals provided appreciated editorial assistance by evaluating manuscripts submitted to the Annals issue. We thank them for their input of time and effort, and for making constructive suggestions which led to improvements in the papers.

Raj Bhansali, University of Liverpool.

Geetesh Bhardwaj, Rutgers University.

Tim Bollerslev, Duke University.

Peter Christoffersen, McGill University.

Valentina Corradi, Queen Mary, University of London.

Alvaro Escribano, University of Carlos III de Madrid.

Gloria Gonzalez, University of California, Riverside.

Graciela Gonzalez-Farias, CIMAT, Mexico.

Niels Haldrup, University of Aarhus.

Bruce Hansen, University of Wisconsin.

David Hendry, Oxford University.

Yongmiao Hong, Cornell University.

Cheng Hsiao, University of Southern California.

Atsushi Inoue, North Carolina State University.

Eric Jacquier, HEC, University of Montréal.

Michael Johannes, Columbia University.

Mark Kamstra, York University. 
Lutz Kilian, University of Michigan.

Tae-Hwy Lee, University of California, Riverside.

Robert Lieli, University of Texas, Austin.

Laura Mayoral, University of Pompeu Fabra.

Serena $\mathrm{Ng}$, University of Michigan.

Jose Olmo, City University, London.

Paolo Parulo, University of Insubria.

Elena Pasavento, Emory University.

Andrew Patton, London School of Economics.

Jean-Yves Pitarakis, University of Southampton.

Jack Porter, University of Wisconsin.

Barbara Rossi, Duke University.

Pedro Santa-Clara, University of California, Los Angeles.

Kevin Shephard, Oxford University.

Anthony Tay, Singapore University.

Timo Teräsvirta, Stockholm University.

Allan Timmerman, University of California, San Diego.

Norman R. Swanson

Department of Economics,

Rutgers University, USA

E-mail address: nswanson@econ.rutgers.edu

Graham Elliott

Department of Economics,

University of California, USA

Eric Ghysels

Departments of Economics and Finance, University of North Carolina, Chapel Hill, USA

Jesus Gonzalo

Department of Economics, Universidad Carlos III de Madrid, Spain 